\title{
OBTENÇÃO DE UM MODELO GEOIDAL PARA O ESTADO DE SÃO PAULO
}

\author{
Msc. Marco Antônio Silva \\ São Paulo - SP - Brasil \\ marcosil@yahoo.com
}

\begin{abstract}
RESUMO
O sistema GPS tem sido largamente usado para posicionamento. Vislumbra-se o potencial uso deste sistema para determinação de altitudes ortométricas, substituindo o oneroso e demorado processo de nivelamento geométrico. Para isso, um modelo geoidal com precisão absoluta submétrica e precisão relativa da ordem de 2 ppm é necessário. Este modelo pode ser dividido em duas componentes: longo e curto comprimento de onda. O modelo do geopotencial fornece a componente de longo comprimento de onda da altura geoidal, enquanto que a gravimetria associada a um modelo digital do terreno permite calcular a componente de curto comprimento de onda através da integral modificada de Stokes. Algumas das modificações da função de Stokes são comparadas. Dois métodos de avaliação desta integral, integração direta e FFT são mostrados no trabalho. Como resultado dessas comparações, obtém-se um modelo geoidal para o Estado de São Paulo.
\end{abstract}

\begin{abstract}
The GPS system has been used broadly for positioning. It is glimpsed the potential use of this system for the determination of orthometric height, substituting the expensive and slow process of geometric leveling. For this propose, it is necessary a geoid model with submetric absolute accuracy and relative accuracy on the order of 2 ppm. This model can be divided in two components: long and short wavelengths. The model of the geopotential supplies the long wavelength component of the geoid height, while gravimetry associated with a digital terrain model, allows the estimation of the short wavelength component, through the modified Stokes's integral. Comparisons of some of these modifications of the Stokes's function are carried out. Two methods of evaluation of the integral, numerical integration and FFT are shown in the work. As a result of those comparisons, it is obtained a geoid model for the State of São Paulo.
\end{abstract}

\section{1 - INTRODUÇÃO}

Existem três superfícies que envolvem o posicionamento geodésico. A primeira é a superfície física limitante do relevo topográfico onde são realizadas as operações topográficas e geodésicas. A segunda é chamada de superfície elipsoidal, limitante de um elipsóide de revolução que é a figura matemática da Terra, gerada pela rotação de uma elipse em torno de seu eixo menor. A terceira é a superfície geoidal que limita um corpo chamado geóide, definida como uma superfície equipotencial do campo de gravidade que mais se aproxima do nível médio não perturbado dos mares, prolongado através dos continentes.

Considerando um ponto sobre a superfície física, a reta que passa por este ponto e é perpendicular ao geóide define a direção de uma linha de força chamada vertical. A reta que passa por este mesmo ponto e é perpendicular ao elipsóide define a direção de outra linha de força chamada normal. A distância entre a superfície física e a superfície 
geoidal, tomada sobre a vertical, é chamada de altitude ortométrica $\mathbf{( H )}$. A distância entre a superfície física e a superfície elipsoidal, tomada sobre a normal, é a altitude geométrica (h). A distância entre a superfície geoidal e a superfície elipsoidal, contado ao longo da normal, é a altura geoidal $(\mathbf{N})$. Esta altura é aproximadamente igual à diferença entre as altitudes ortométrica e geométrica. Diz-se aproximadamente porque as retas suporte dessas altitudes não são colineares.

$$
\mathrm{N} \cong \mathrm{H}-\mathrm{h}
$$

A altitude ortométrica é obtida através do nivelamento geométrico associado a medições adequadas da aceleração da gravidade. A obtenção da altitude ortométrica exige o conhecimento da gravidade média entre as superfícies física e geoidal, o que é impossível devido ao não conhecimento da distribuição de densidade de massa no interior da crosta terrestre. Uma alternativa é utilizar um valor conveniente da gravidade da Terra normal, obtendo-se a altitude normal.

A altitude geométrica é obtida diretamente através do GPS. Como o processo do nivelamento geométrico é dispendioso e muito demorado e o GPS tem sido largamente usado para posicionamento, vislumbra-se o potencial uso do GPS para a determinação de altitudes ortométricas. Para tanto é necessário que exista um modelo de alturas geoidais para a região de interesse.

O desenvolvimento dos modelos geoidais tomou um grande impulso com o advento dos satélites artificiais. Estes funcionam como sensores do campo gravitacional e a análise das perturbações de suas órbitas possibilitou o aprimoramento do cálculo dos coeficientes do desenvolvimento em série do modelo do geopotencial. $\mathrm{O}$ Earth Geopotential Model 1996 - EGM96 é o mais recente modelo publicado e foi usado neste trabalho.

O modelo geoidal pode ser dividido em duas componentes: longo e curto comprimento de onda. O modelo do geopotencial fornece a componente de longo comprimento de onda da altura geoidal, enquanto que a gravimetria associada a um modelo digital do terreno, permite calcular a componente de curto comprimento de onda, através da integral modificada de Stokes.

A integral de Stokes que durante muito tempo foi considerada como teórica passou a ser facilmente aplicável a partir dos modelos do geopotencial, os quais permitiram estender a integral, ao invés da toda a Terra, a uma vizinhança do ponto de cálculo. Com isso, algumas modificações na função de Stokes foram pesquisadas. As modificações propostas por P. Vanicek e L. E. Kleusberg (1987), L. Wong e R. Gore (1969) e W. E. Featherstone, J. D. Evans e J. G. Olliver (1998), denominadas resumidamente aqui de Vanicek-Kleusberg, Wong-Gore e Featherstone-Vanicek, respectivamente, são apresentadas e comparadas neste trabalho.

O método tradicional de resolução da integral de Stokes é a integração numérica. O inconveniente deste método é o tempo muito grande de computação. Aplicando a Transformada de Fourier a integral de Stokes se transforma numa simples multiplicação de funções, uma vez tratar-se de uma integral de convolução, o que diminui o tempo de computação, mas exige que os pontos de cálculo estejam dispostos em uma malha regular. Os métodos, integração numérica e Transformada de Fourier são comparados neste trabalho. 


\section{2 - DETERMINAÇÃO DO GEÓIDE}

\section{1 - A Integral de Stokes}

George Gabriel Stokes apresentou em 1849, uma das fórmulas mais importantes da Geodésia Física, pois, torna possível determinar a separação geóide-elipsóide, ou seja, a ondulação geoidal, através do conhecimento das anomalias da gravidade. Ela foi considerada durante muito tempo como apenas teórica, pois necessitava do conhecimento da gravidade em toda a extensão da Terra, inclusive nos oceanos, o que era tido como impossível. Com o desenvolvimento tecnológico, principalmente o uso de satélites artificiais para determinar os modelos do geopotencial, a integral não precisa mais ser estendida a toda a Terra, e sim a uma vizinhança do ponto de cálculo. Para isso é necessário introduzir uma modificação na mesma integral. Foram propostas várias modificações sendo que algumas delas serão apresentadas e comparadas neste trabalho. A componente de longo comprimento de onda da altura geoidal é baseada nos modelos do geopotencial.

$\mathrm{Na}$ dedução da fórmula, Stokes admitiu a inexistência de massas externas à superfície equipotencial considerada, o que faz com que se deva reduzir as medições da gravidade feitas na superfície física para o geóide e compensar as massas que estão externas ao geóide. Também admitiu uma aproximação esférica para a superfície da Terra.

A integral de Stokes tem a seguinte forma:

$$
\mathrm{N}=\frac{\mathrm{R}}{4 \pi \gamma} \int_{0}^{\pi} \int_{0}^{2 \pi} \Delta \mathrm{g} \mathrm{S}(\psi) \operatorname{sen} \psi \mathrm{d} \psi \mathrm{d} \alpha
$$

onde $\mathbf{N}$ é a altura geoidal, $\mathbf{R}$ é o raio da Terra, $\boldsymbol{\gamma}$ é o valor médio da gravidade da Terra normal, $\Delta \mathbf{g}$ é a anomalia da gravidade, $(\boldsymbol{\psi}, \boldsymbol{\alpha})$ distância e azimute esférico entre o ponto considerado e o ponto onde $\mathbf{N}$ é calculado e $\mathbf{S}(\boldsymbol{\Psi})$ é a função de Stokes.

A função $\mathbf{S}(\boldsymbol{\Psi})$ pode ser expressa em uma expansão em série de polinômios de Legendre da seguinte forma:

$$
S(\psi)=\sum_{n=2}^{\infty} \frac{2 n+1}{n-1} P_{n}(\cos \psi)
$$

onde $\mathbf{P}_{\mathbf{n}}$ são os polinômios de Legendre de grau $\mathbf{n}$.

Como já foi mencionado, a Integral de Stokes pressupõe a não existência de massas externas ao geóide. Para isto reduzem-se as medições da gravidade, feitas sobre a superfície física, para o geóide e eliminam-se as massas topográficas. Com essas reduções, cria-se uma Terra fictícia com características diferentes da Terra real. Em seguida, através da Integral de Stokes determina-se a separação entre o elipsóide de referência e um geóide fictício, que é o corpo resultante da Terra modificada. Esse geóide fictício é chamado de cogeóide. Para cada método de remoção das massas topográficas resulta em um cogeóide. Tem-se então mais uma grandeza a determinar que é a separação entre o geóide e o cogeóide. Essa separação é conhecida como efeito indireto. $\mathrm{O}$ que se procura é adotar um método de remoção das massas topográficas que resulte no menor efeito indireto possível.

A avaliação da integral pode ser feita de duas maneiras: por integração numérica ou por FFT - Fast Fourier Transform. Os dois métodos de avaliação são comparados neste trabalho. A avaliação da integral através de FFT foi a metodologia usada para estimar as ondulações geoidais para o Estado de São Paulo. 


\section{2 - Os Modelos do Geopotencial}

Um modelo do geopotencial é obtido determinando-se os valores para os coeficientes $A_{n m}$ e $B_{n m}$ na expressão do potencial de gravidade, até um dado grau e ordem. Os satélites artificiais trouxeram uma contribuição significativa na obtenção destes modelos para os coeficientes de grau e ordem mais baixos.

O fato que leva à utilização dos satélites é que seus movimentos estão sujeitos a perturbações devido à não homogeneidade na distribuição de massa da Terra. $\mathrm{O}$ estudo destas perturbações permite modelar as heterogeneidades, as quais se relacionam aos valores dos coeficientes acima citados. A contribuição dos satélites se restringe aos coeficientes de ordem mais baixa, pois eles são influenciados em seu movimento por variações de longo comprimento de onda do campo gravitacional.

A determinação dos coeficientes de ordem mais elevada exige o conhecimento das variações de curto comprimento de onda, que só podem ser obtidos por observações sobre a superfície. Os modelos mais modernos, como o EGM96 que foi usado neste trabalho, atingem o grau e ordem 360.

O modelo do geopotencial representa a superfície denominada de esferóide, a qual é intermediária entre o geóide e o elipsóide.

A equação da altura geoidal $\mathrm{N}$ pode ser escrita de forma abreviada:

$$
\mathrm{N}(\theta, \lambda)=\mathrm{N}_{\mathrm{LC}}(\theta, \lambda)+\mathrm{N}_{\mathrm{CC}}(\theta, \lambda)
$$

Isto significa dividir a altura geoidal em duas componentes espectrais: uma componente de longo comprimento de onda $\mathbf{N}_{\mathbf{L C}}$ e uma de curto comprimento de onda $\mathbf{N}_{\mathbf{C C}}$. A primeira representa a separação entre o elipsóide de referência e o esferóide representado pelo modelo do geopotencial. A segunda representa a separação entre o esferóide e o geóide.

A primeira componente é calculada através do modelo do geopotencial, o que significa estender a série até um dado grau e ordem L. A segunda componente é determinada através das anomalias da gravidade por meio de uma modificação da Integral de Stokes.

\section{3 - Integral Modificada de Stokes}

Com a possibilidade do uso de um modelo do geopotencial para o cálculo da componente de longo comprimento de onda da altura geoidal, não é mais necessário estender a Integral de Stokes a toda a Terra, eliminando assim a limitação de uso da mesma. Pode-se aplicar a integral a uma região próxima ao ponto de cálculo, limitada por um raio $\Psi_{\mathbf{0}}$, que é a distância esférica máxima de aplicação da integral. Mesmo que a integral seja calculada em toda a área limitada pelo raio $\boldsymbol{\psi}_{\mathbf{0}}$, isto representa uma aproximação da Integral de Stokes. Como resultado desta limitação tem-se um erro de truncamento da integral. A partir do raio $\boldsymbol{\psi}_{0}$ o cálculo é feito através dos modelos do geopotencial o que minimiza ou quase anula o erro de truncamento. Por isso este erro é comumente negligenciado durante determinações práticas de modelos geoidais, particularmente quando se usa a FFT como método de avaliação da integral (Schwarz et al., 1990). Para reduzir o erro de truncamento é necessária uma modificação na integral original de Stokes que consiste em modificar a função de Stokes $\mathbf{S}(\boldsymbol{\psi})$ e a anomalia da gravidade. Diversas propostas de modificação da função de Stokes têm sido apresentadas. Neste trabalho são mostradas e comparadas as modificações propostas por P. Vanicek e L. E Kleusberg (1987), L. Wong e R. Gore (1969) e P. Vanicek e W. E. Featherstone (1998), denominadas resumidamente aqui de Vanicek-Kleusberg, WongGore e Featherstone-Vanicek, respectivamente.

A Integral de Stokes modificada pode ser escrita da seguinte maneira: 


$$
\mathrm{N}^{\mathrm{M}}(\theta, \lambda)=\frac{\mathrm{R}}{4 \pi \gamma} \int_{0}^{\psi_{0}} \int_{0}^{2 \pi} \Delta \mathrm{g}(\alpha, \psi) \mathrm{S}^{\mathrm{M}}(\psi) \operatorname{sen} \psi \mathrm{d} \psi \mathrm{d} \alpha
$$

sendo $S^{\mathrm{M}}(\psi)$ a função modificada de Stokes.

L. Wong e R. Gore (1969) propuseram uma modificação baseada na remoção dos polinômios de grau mais baixo da função de Stokes, reduzindo com isso o erro de truncamento. Esta modificação é dada por:

$$
\mathrm{S}_{\mathrm{wg}}^{\mathrm{M}}(\psi)=\mathrm{S}(\psi)-\sum_{\mathrm{n}=2}^{\mathrm{M}-1} \frac{2 \mathrm{n}+1}{\mathrm{n}-1} \mathrm{P}_{\mathrm{n}}(\cos \psi)
$$

onde $\mathbf{P}_{\mathbf{n}}(\cos \psi)$ são os polinômios de Legendre de grau $\mathbf{n}$ e $\mathbf{M}$ é o grau, até o qual, será removido da função de Stokes. Experimentos feitos por Featherstone e Sideris (1997) usaram $\mathbf{M}=\mathbf{3 6}$ e $\mathbf{M}=\mathbf{3 6 0}$ para esta modificação. A modificação de Wong-Gore quando usada em determinações de modelos regionais do geóide funciona como um filtro passaalta.

P. Vanicek e L. E. Kleusberg (1987) propuseram uma modificação na função de Stokes visando minimizar o limite superior do erro de truncamento em termos de desvio padrão. Esta modificação pode ser expressa assim (Featherstone et al (1998):

$$
\mathrm{S}_{\mathrm{vk}}^{\mathrm{M}}(\psi)=\mathrm{S}^{\mathrm{M}}(\psi)-\sum_{\mathrm{n}=2}^{\mathrm{M}-1} \frac{2 \mathrm{k}+1}{2} \mathrm{t}_{\mathrm{k}}\left(\psi_{0}\right) \mathrm{P}_{\mathrm{k}}(\cos \psi)
$$

onde $t_{k}$ são os coeficientes da modificação. Segundo Vanicek e Sjoberg (1991) esta modificação reduz mais o erro de truncamento do que a modificação de Wong-Gore.

P. Meissl (1971) propôs uma modificação simples para a função de Stokes. Subtrair da função original $\mathbf{S}(\boldsymbol{\psi})$ o valor numérico da função no raio de truncamento $\mathbf{S}\left(\boldsymbol{\Psi}_{\mathbf{0}}\right)$. Esta modificação é dada por:

$$
\mathrm{S}_{\mathrm{me}}(\psi)=\mathrm{S}(\psi)-\mathrm{S}\left(\psi_{0}\right)
$$

Esta modificação faz com que a série do erro de truncamento convirja para zero mais rapidamente, principalmente quando usada em conjunto com a técnica de removecalcula-restaura (Featherstone et al (1998).

Featherstone W. E., Evans J. D. e Olliver J. G. (1998) propuseram uma modificação híbrida, combinando a modificação de P. Meissl com a de Vanicek e Kleusberg. Eles aplicam a modificação de Miessl, usando como função, a função modificada de Vanicek e Kleusberg. Este procedimento reduz ainda mais o erro de truncamento do que se as modificações fossem aplicadas separadamente. Ela pode ser assim expressa:

$$
\mathrm{S}_{\mathrm{mvk}}^{\mathrm{M}}(\psi)=\mathrm{S}_{\mathrm{vk}}^{\mathrm{M}}(\psi)-\mathrm{S}_{\mathrm{vk}}^{\mathrm{M}}\left(\psi_{0}\right)
$$

Para esta modificação os coeficientes de truncamento $\mathrm{Q}_{\mathrm{n}}^{\prime \mathrm{m}^{*}}\left(\psi_{0}\right)$ são determinados com segue:

$$
\mathrm{K}_{\mathrm{mvk}}^{\mathrm{M}}\left(\psi, \psi_{0}\right)=\left\{\begin{array}{lll}
\mathrm{S}_{\mathrm{vk}}^{\mathrm{M}}\left(\psi_{0}\right) & \text { para } & 0 \leq \psi \leq \psi_{0} \\
\mathrm{~S}_{\mathrm{vk}}^{\mathrm{M}}(\psi) & \text { para } \psi_{0} \leq \psi \leq \pi
\end{array}\right.
$$

Para o produto $\Delta \mathrm{g} \mathrm{S}^{\mathrm{M}}$ tornar-se compatível com o grau e ordem do modelo do geopotencial utilizado é necessário que a anomalia da gravidade seja subtraída dos harmônicos de grau e ordem mais baixos. Assim a anomalia da gravidade modificada é calculada com: 


$$
\Delta \mathrm{g}_{\mathrm{L}}^{\mathrm{M}}=\Delta \mathrm{g}-\sum_{\mathrm{n}=0}^{\mathrm{L}} \sum_{\mathrm{m}=0}^{\mathrm{n}}\left(\mathrm{C}_{\mathrm{nm}} \cos \mathrm{m} \lambda+\mathrm{D}_{\mathrm{nm}} \operatorname{sen} \mathrm{m} \lambda\right) \mathrm{P}_{\mathrm{nm}} \cos \theta
$$

Com essa modificação, a expressão final para a integral de Stokes fica:

$$
\mathrm{N}^{\mathrm{M}}(\theta, \lambda)=\frac{\mathrm{R}}{4 \pi \gamma} \int_{0}^{\psi_{0}} \int_{0}^{2 \pi} \Delta \mathrm{g}^{\mathrm{M}}(\alpha, \psi) \mathrm{S}^{\mathrm{m}}(\psi) \operatorname{sen} \psi \mathrm{d} \psi \mathrm{d} \alpha
$$

Como a influência dos longos comprimentos de onda é levada em consideração pelo modelo do geopotencial, a função de Stokes fica limitada a uma vizinhança $\boldsymbol{\Psi}_{\mathbf{0}}$.

A altura geoidal se completa com a componente de longo comprimento de onda $\mathbf{N}_{\mathbf{L C}}$ calculada através dos coeficientes do modelo do geopotencial até o grau e ordem $\mathbf{L}$. Daí a justificativa para a expressão remove-calcula-restaura.

\section{3 - DADOS UTILIZADOS PARA O CÁLCULO DO SPGEOID2002}

Os dados usados para cálculo do SPGEOID2002 são provenientes de várias fontes. O GETECH da Universidade de Leeds, Inglaterra, tem desde 1986, reunido e organizado os dados gravimétricos de diversos continentes. Na América do Sul foi completado em 1990 o SAGP - South American Gravity Project que no Brasil cobriu algumas áreas e revelou outras com poucas informações gravimétricas, como o Centro Oeste e Norte do país. A EPUSP e o IBGE realizaram um esforço conjunto na última década cobrindo parte considerável destas áreas. Além desta iniciativa faz parte do acervo de dados gravimétricos da EPUSP usados no cálculo do SPGEOID2002, dados provenientes de empresas públicas e privadas que executam levantamentos gravimétricos no Brasil, entre elas a Petrobrás, Companhia de Pesquisas de Recursos Minerais e o Observatório Nacional. É de muita importância a contribuição dada pelo IAG-USP, através do Departamento de Geofísica. Alguns dados são provenientes de universidades que também realizam pesquisas na área de Geodésia e compartilham dados gravimétricos, como a Universidade Federal do Paraná, Universidade de Brasília, Universidade de Manaus.

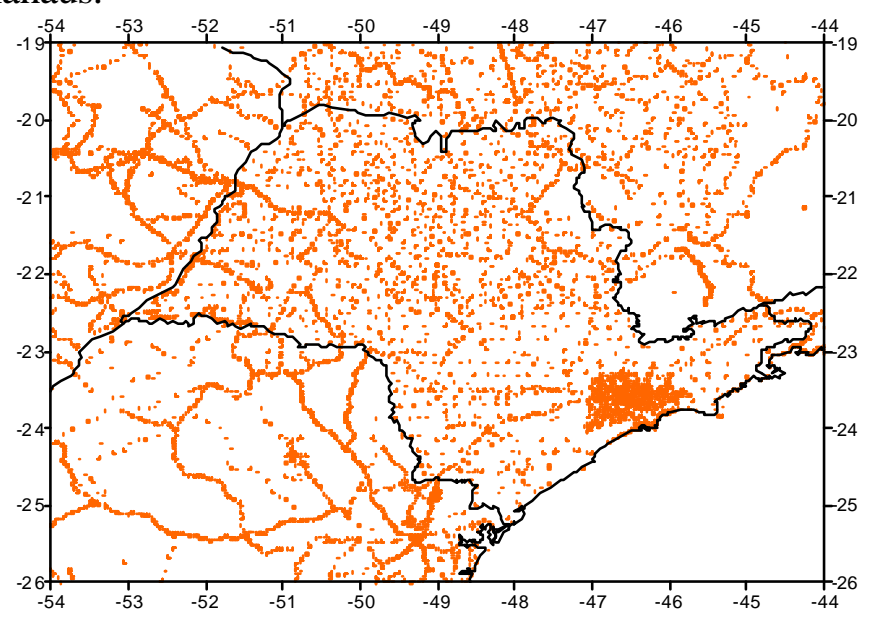

Figura 3.1 - Distribuição dos dados gravimétricos no Estado de São Paulo

\section{2 - Dados Altimétricos}

Um modelo digital do terreno confiável é de fundamental importância para a gravimetria. Dele, dependem as correções aplicadas aos valores de gravidade medidos 
sobre a superfície física para valores sobre a superfície geoidal. Por isso o SAGP criou um modelo digital do terreno para toda a América do Sul consistindo de um modelo de altitudes para o continente e de profundidades para os oceanos numa grade com espaçamento de 3'.

Para definição deste modelo, foram utilizados vários tipos de informações topográficas:

- Modelo de altitudes global denominado ETOPO5;

- Valores digitalizados a partir de cartas topográficas;

- Alturas estabelecidas nas estações gravimétricas;

- Locação de linhas costeiras.

A Figura 3.2 mostra o modelo digital do terreno usado no cálculo do SPGEOID2002 no Estado de São Paulo.

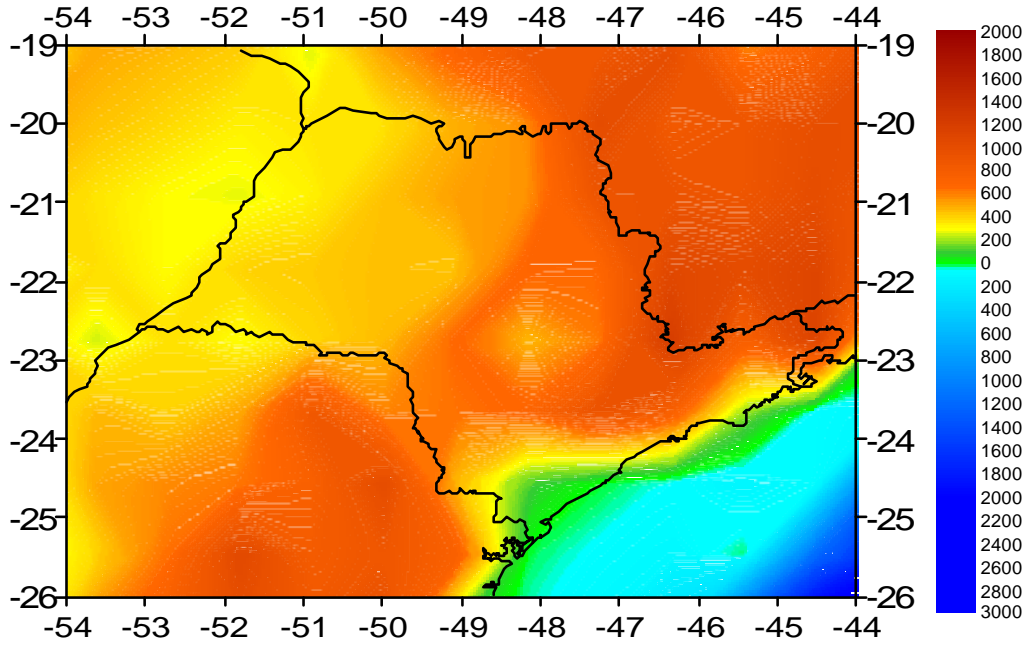

Figura 3.2 - Modelo Digital do Terreno para o Estado São Paulo

\section{3 - Processamentos dos Dados do SPGEOID2002}

O LTG-EPUSP possui um acervo de dados e programas para cálculos geoidais que vem sendo utilizado em outras pesquisas e atividades da escola.

Do arquivo geral de dados gravimétricos da América do Sul foi gerado um arquivo de anomalias médias para quadrículas de 5'x 5'.

Verificou-se a presença de muitas áreas vazias, que não possuíam valores médios de anomalias decorrentes da falta de cobertura gravimétrica. Então optou-se por gerar um arquivo de anomalias médias para quadrículas de $10^{\prime}$ x $10^{\prime}$ derivado das anomalias médias de $5^{\prime} \times 5^{\prime}$.

Os modelos digitais do terreno e correção do relevo foram também processados e obtidos valores médios para as mesmas quadrículas.

Para as regiões oceânicas que faziam parte da área foi usado o modelo global de anomalias da gravidade para o oceano, denominado KMS99. Este modelo foi calculado com dados dos satélites altimétricos ERS-1 e GEOSAT e publicado pelo Kort \& Matrikstyrelsen da Dinamarca. O modelo é formado por valores de anomalias da gravidade, em mGal, numa grade regular com resolução de $2^{\prime}$ x $2^{\prime}$, que cobre as áreas oceânicas.

O desenvolvimento completo deste modelo pode ser encontrado Andersen O; Knudsen P.; Trimmer R. (2000). Estes dados são de domínio público e estão disponíveis endereço de FTP do KMS.

Para avaliar o geóide calculado foi usado um arquivo de 175 pontos da rede de nivelamento onde foram feitas observações com satélites de posicionamento, 86 pontos 
com o antigo sistema TRANSIT, denominados de pontos DOP e 89 pontos com o sistema GPS, denominados de pontos GPS.

Foram usados basicamente três softwares para o processamento do modelo geoidal SPGEOID2002. Dois desenvolvidos pelo Prof. Denizar Blitzkow que foi usado para executar os cálculos e avaliações do modelo EGM96 e para executar o cálculo da integral de Stokes pelo método da integração numérica. Para executar o cálculo do pelo método da FFT foi usado o software desenvolvido pelo Prof. M. Sideris e modificado por W. E. Featherstone que implementou as rotinas de cálculo para as diferentes modificações da função de Stokes.

\section{4 - RESULTADOS E DISCUSSÃO}

\section{1 - Avaliação do Modelo do Geopotencial EGM96}

Primeiramente foi feita uma avaliação do modelo EGM96, comparando os valores das alturas esferoidais obtidas através do modelo com alturas geoidais obtidas através de satélites de posicionamento. Foram utilizados os 175 pontos, 86 pontos DOP e 89 pontos GPS. Estes pontos fazem parte da Rede Brasileira de Nivelamento do IBGE, onde foram feitas observações de satélites. A avaliação foi feita para diferentes valores de grau e ordem do modelo do geopotencial: $36,50,72,100,180$ e 360 . O sistema utilizado para as comparações foi o WGS84.

Para cada ponto foi obtida a diferença entre a altura geoidal por satélites $\mathbf{N}_{\mathrm{s}}$ e a altura esferoidal obtida pelo modelo EGM96 $\mathbf{N}_{\mathbf{E}}$.

$$
\Delta \mathrm{N}=\mathrm{N}_{\mathrm{S}}-\mathrm{N}_{\mathrm{E}}
$$

A partir das diferenças foi obtida a diferença padrão $\sigma_{\Delta \mathbf{N}}$ :

$$
\sigma_{\Delta N}=\sqrt{\frac{\Sigma(\Delta N)^{2}}{n(n-1)}}
$$

sendo $\mathbf{n}$ o número de pontos disponíveis.

A média das diferenças é a chamada altura geoidal de ordem zero $\mathbf{N}_{\mathbf{0}}$ que representa a diferença do semi-eixo maior entre o elipsóide de referência WGS84 e o elipsóide que melhor se adapta ao modelo do geopotencial EGM96 (Blitzkow; Sá, 1983). Os resultados desta verificação estão na Tabela 4.1.

\begin{tabular}{|c|c|c|c|c|c|c|c|c|}
\hline \multicolumn{7}{|c|}{ Resumo dos Resultados da Avaliação do EGM96 } \\
\hline $\begin{array}{c}\text { Grau e } \\
\text { Ordem }\end{array}$ & \multicolumn{2}{|c|}{$\begin{array}{c}\text { Diferença } \\
\text { Padrão (m) }\end{array}$} & \multicolumn{2}{c|}{$\begin{array}{c}\text { Altura Geoidal de } \\
\text { ordem zero } \mathbf{N}_{\mathbf{0}}(\mathbf{m})\end{array}$} & \multicolumn{2}{c|}{$\begin{array}{c}\text { Valor } \\
\text { Máximo (m) }\end{array}$} & \multicolumn{2}{c|}{$\begin{array}{c}\text { Valor } \\
\text { Mínimo (m) }\end{array}$} \\
\hline & DOP & GPS & DOP & GPS & DOP & GPS & DOP & GPS \\
\hline $\mathbf{3 6}$ & 1,37 & 1,75 & $-0,66$ & $-0,78$ & 4,35 & 4,14 & $-5,39$ & -2.69 \\
\hline $\mathbf{5 0}$ & 1,13 & 0,71 & $-0,05$ & $-0,15$ & 1,93 & 1,58 & $-2,94$ & $-3,41$ \\
\hline $\mathbf{7 2}$ & 1,05 & 0,71 & $-0,56$ & $-0,34$ & 2,01 & 1,97 & $-3,31$ & $-2,67$ \\
\hline $\mathbf{1 0 0}$ & 1,16 & 0,70 & $-0,77$ & $-0,36$ & 1,70 & 2,51 & $-3,08$ & $-3,18$ \\
\hline $\mathbf{1 8 0}$ & 1,12 & 0,62 & $-0,70$ & $-0,30$ & 1,69 & 2,60 & $-3,23$ & $-2,93$ \\
\hline $\mathbf{3 6 0}$ & 1,07 & 0,79 & $-0,65$ & $-0,49$ & 1,64 & 2,71 & 3,63 & $-3,73$ \\
\hline
\end{tabular}

Tabela 4.1 - Comparação entre os pontos DOP, GPS e o modelo EGM96

Analisando os dados da Tabela 4.1, verifica-se que a diferença padrão, aplicação de expressão (4-2), para os pontos DOP ficou em torno 1,10 m, com exceção do grau e ordem 36 que está em 1,37 m. Para os pontos GPS a diferença padrão está em torno de $0,70 \mathrm{~m}$, menor $0,40 \mathrm{~m}$ do que os pontos DOP, com exceção do grau e ordem 36 que esta 
em $1,75 \mathrm{~m}$. Verifica-se também que as diferenças padrão para os graus 50, 72, 100, 180 e 360 diferem entre si de aproximadamente $0,17 \mathrm{~m}$. Este fato nos mostra que acima do grau e ordem 50 as diferenças padrão se mantêm. Os valores das diferenças diminuem à medida que aumenta o grau e ordem do modelo do geopotencial, até o grau 180. A partir deste, as diferenças aumentam, chegando a 0,79 para o grau 360. Este aumento é devido à falta de dados gravimétricos que influenciariam nos graus e ordem mais altos, quando do cálculo do modelo EGM96. Com relação à $3^{\circ}$ coluna $\left(\mathbf{N}_{\mathbf{0}}\right)$, a média nas diferentes estimativas é de $-0,56 \mathrm{~m}$ para os pontos DOP e $-0,40 \mathrm{~m}$ para os pontos GPS, ou seja, o elipsóide que mais se adapta ao modelo avaliado, é um elipsóide com semieixo maior de aproximadamente $6378136,50 \mathrm{~m}$, menor $0,50 \mathrm{~m}$ do que o elipsóide WGS84 usado na avaliação. Todos os histogramas mostram tendência de distribuição não normal com média das diferenças diferente de zero, significando a existência de alguma diferença sistemática nos dados.

\section{2 - Cálculo do Modelo Geoidal}

Após a avaliação do modelo EGM96 através dos pontos DOP e GPS, passou-se ao cálculo das alturas geoidais utilizando, além do modelo do geopotencial, dados de anomalias médias da gravidade e modelos digitais do terreno nos continentes e de altimetria por radar nas regiões oceânicas.

O procedimento matemático usado para calcular o SPGEOID2002 é conhecido como remove-calcula-restaura. Ou seja, removeu-se das anomalias médias da gravidade a componente de longo comprimento de onda, dada pelo modelo do geopotencial EGM96. Optou-se pelo grau e ordem 50. Calculou-se a altura geoidal através da integral modificada de Stokes estendendo-se a uma vizinhança $\boldsymbol{\psi}_{\mathbf{0}}=\mathbf{4}^{\mathbf{0}}$. Em seguida restaurou-se na altura geoidal a componente de longo comprimento de onda, através do modelo do geopotencial EGM96, evidentemente no mesmo grau e ordem.

Existem dois procedimentos para avaliação da integral de Stokes: a integração numérica e o FFT. A vantagem do FFT é o menor tempo de processamento em relação ao método de integração numérica. Foi executado o cálculo pelos dois procedimentos acima citados, sendo feitos diferentes experimentos, atribuindo-se as seguintes denominações:

- INT-VK - Integração numérica da fórmula Stokes, com a modificação de Vanicek-Kleusberg, modificação descrita pela expressão (2-6);

- FFT-FV - Integral de Stokes, por FFT com a modificação de FeatherstoneVanicek, modificação descrita pela expressão (2-8);

- FFT-WG - Integral de Stokes, por FFT com a modificação de Wong-Gore, modificação descrita pela expressão (2-5);

- FTT-VK - Integral de Stokes, por FFT com a modificação de VanicekKleusberg, modificação descrita pela expressão (2-6).

Os cálculos foram feitos numa malha regular com espaçamento de 10'x10' num retângulo que envolve o Estado de São Paulo. A malha contém 162 linhas por 180 colunas, sendo que os pontos coincidem com o ponto central da quadrícula.

Os modelos geoidais decorrentes dos quatro experimentos foram comparados entre si para verificar a consistência dos resultados. Estes apresentados na Tabela 4.2. 


\begin{tabular}{|c|c|c|c|c|}
\hline \multicolumn{6}{|c|}{ Resumo das comparações entre os experimentos FFT } \\
\hline Experimentos & $\begin{array}{c}\text { Diferença } \\
\text { Padrão (m) }\end{array}$ & $\begin{array}{c}\text { Média das } \\
\text { Diferenças (m) }\end{array}$ & $\begin{array}{c}\text { Valor } \\
\text { Máximo (m) }\end{array}$ & $\begin{array}{c}\text { Valor } \\
\text { Mínimo (m) }\end{array}$ \\
\hline FFT-FV e FFT-VK & 0,01 & 0,01 & 0,02 & $-0,01$ \\
\hline FFT-WG e FFT-VK & 0,32 & $-0,24$ & 0,86 & $-0,19$ \\
\hline FFT-FV e FFT-WG & 0,32 & $-0,24$ & 0,84 & $-0,19$ \\
\hline
\end{tabular}

Tabela 4.2 - Resumo das comparações entre os experimentos FFT

Como pode ser verificado na tabela acima, o resultado do cálculo através das modificações de Vanicek-Kleusberg e Featherstone-Vanicek, experimentos FFT-VK e FFT-FV, produziram resultados muito semelhantes, ou seja, a diferença padrão ficou entre $-0,01 \mathrm{~m}$ e $0,02 \mathrm{~m}$ com média de $0,01 \mathrm{~m}$. Já a modificação de Wong-Gore, experimento FFT-WG, produziu resultado bastante diferente em relação aos dois citados acima, com as diferenças entre $-0,19 \mathrm{~m}$ a $0,86 \mathrm{~m}$, com diferença padrão de 0,32 $\mathrm{m}$. A média das diferenças ficou em $0,24 \mathrm{~m}$, significando a existência de alguma diferença sistemática nos dados comparados.

A Tabela 4.3 se refere à comparação dos cálculos feitos com a integral de Stokes avaliada pelo método da integração numérica, experimento INT-VK, e os resultados feitos por FFT, com cada uma das modificações já citadas, experimentos FFT-VK, FFT-FV e FFT-WG.

\begin{tabular}{|c|c|c|c|c|}
\hline \multicolumn{6}{|c|}{ Resumo das comparações entre os experimentos INT e FFT } \\
\hline Experimento & $\begin{array}{c}\text { Diferença } \\
\text { Padrão (m) }\end{array}$ & $\begin{array}{c}\text { Média das } \\
\text { Diferenças (m) }\end{array}$ & $\begin{array}{c}\text { Valor } \\
\text { Máximo (m) }\end{array}$ & $\begin{array}{c}\text { Valor } \\
\text { Mínimo (m) }\end{array}$ \\
\hline INT-VK e FFT-VK & 0,54 & 0,01 & 1,88 & $-1,52$ \\
\hline INT-VK e FFT-FV & 0,54 & 0,01 & 1,88 & $-1,52$ \\
\hline INT-VK e FFT-WG & 0,63 & $-0,23$ & 1,69 & $-1,93$ \\
\hline
\end{tabular}

Tabela 4.3 - Resumo das comparações entre os experimentos INT e FFT

Como pode ser constatado nos resultados das comparações entre as modificações de Vanicek-Kleusberg e Featherstone-Vanicek calculadas via FFT e Vanicek-Kleusberg via integração numérica, a diferença padrão e a média das diferenças são as mesmas $0,54 \mathrm{~m}$ e $0,01 \mathrm{~m}$ respectivamente, pois estas modificações são muito semelhantes. A média das diferenças em $0.01 \mathrm{~m}$ significa a não existência de diferença sistemática. O resultado da modificação de Wong-Gore via FFT resultou numa diferença padrão e média das diferenças, maiores que as anteriores $0,63 \mathrm{~m} \mathrm{e}-0,23$ $\mathrm{m}$, respectivamente. Este último significando alguma diferença sistemática.

Para avaliação da exatidão das alturas geoidais calculadas foram usados 86 pontos DOP e 89 pontos GPS, cujas alturas geoidais serão consideradas como referência. $\mathrm{O}$ resultado deste procedimento está na Tabela 4.4. 


\begin{tabular}{|c|c|c|c|c|c|c|c|c|}
\hline \multicolumn{1}{|c|}{ Resumo das comparações entre os experimentos e os pontos DOP e GPS } \\
\hline Experimento & $\begin{array}{c}\text { Diferença } \\
\text { Padrão (m) }\end{array}$ & \multicolumn{2}{c|}{$\begin{array}{c}\text { Média das } \\
\text { Diferenças (m) }\end{array}$} & \multicolumn{2}{c|}{$\begin{array}{c}\text { Valor } \\
\text { Máximo (m) }\end{array}$} & \multicolumn{2}{c|}{$\begin{array}{c}\text { Valor } \\
\text { Mínimo (m) }\end{array}$} \\
\hline & DOP & GPS & DOP & GPS & DOP & GPS & DOP & GPS \\
\hline INT-VK & 1,17 & 0,70 & $-0,58$ & $-0,49$ & 1,38 & 1,33 & 3,20 & $-1,65$ \\
\hline FFT-VK & 1,06 & 0,78 & $-0,52$ & $-0,74$ & 1,36 & $-0,04$ & $-3,70$ & $-1,57$ \\
\hline FFT-FV & 1,05 & 0,77 & $-0,51$ & $-0,74$ & 1,37 & $-0,04$ & $-3,69$ & $-1,56$ \\
\hline FFT-WG & 0,98 & 0,62 & $-0,21$ & $-0,46$ & 1,77 & 0,29 & $-3,45$ & $-1,47$ \\
\hline
\end{tabular}

Tabela 4.4 - Resumo das comparações entre experimentos e os pontos DOP e GPS

Analisando a Tabela 4.4 verifica-se que, para todos os experimentos, as diferenças padrão para os pontos DOP foram sempre maiores do que para os pontos GPS. Este resultado já era esperado, pois, independente da distribuição não ser a ideal, sabe-se que os pontos GPS são mais precisos do que os pontos DOP.

Verifica-se também que o experimento FFT-WG resultou numa menor diferença padrão $0,62 \mathrm{~m}$ e média das diferenças $-0,46 \mathrm{~m}$, se comparado com os outros experimentos. Mais uma vez a média das diferenças mostra alguma diferença sistemática nos dados.

Analisando a Tabela 4.4 verifica-se que, para todos os experimentos, as diferenças padrão para os pontos DOP foram sempre maiores do que para os pontos GPS, resultado semelhante ao encontrado para a avaliação do EGM96.

Verifica-se também que o experimento FFT-WG resultou numa menor diferença padrão $0,62 \mathrm{~m}$ e média das diferenças $-0,46 \mathrm{~m}$ se comparado com os outros experimentos, ou seja, o modelo geoidal calculado através da FFT com a modificação da função de Stokes proposta por L. Wong e R. Gore resultou em um geóide com menor diferença quando comparado com os pontos DOP e GPS. As diferenças padrão e a média das diferenças para os experimentos FFT-VK e FFT-FV são praticamente as mesmas. Já para o experimento INT-VK os valores foram menores. Analisando as médias das diferenças, nota-se que a diferença sistemática no método de integração numérica (INT-VK) é 0,25 m menor do que no método de FFT.

\section{5 - CONCLUSÕES}

Com relação à avaliação do EGM96 para o Estado de São Paulo, para certas aplicações onde o modelo geoidal com precisão absoluta em torno de $1,0 \mathrm{~m}$ seja aceitável, pode-se usar somente o modelo do geopotencial. Quando se deseja um modelo geoidal com erros menores do que $1,0 \mathrm{~m}$ é necessário combinar o modelo do geopotencial com dados de gravimetria;

As diferenças para os pontos DOP e GPS, quando se utilizou somente o modelo do geopotencial EGM96 e se quando combinou este modelo com as anomalias médias da gravidade, não se diferenciaram muito. Isto pode ser observado em regiões que, como o Estado de São Paulo, tem a superfície geoidal levemente ondulada. Para regiões onde a superfície geoidal se apresenta mais irregular, este fato não deve ocorrer.

Os cálculos através das modificações de Vanicek-Kleusberg e FeatherstoneVanicek, experimentos FFT-VK e FFT-FV, produziram resultados muito semelhantes, pois, a modificação Featherstone-Vanicek é uma modificação híbrida entre a VanicekKleusberg e Meissl, portanto, era esperado que seus resultados fossem muito parecidos;

A remoção até o grau e ordem 50 para o modelo do geopotencial EGM96 e a extensão da integral de Stokes até o raio de integração $\boldsymbol{\psi}_{\mathbf{0}}=\mathbf{4}^{\mathbf{0}}$ foram valores definidos de maneira, até certo ponto empírica. Sabe-se que o modelo do geopotencial EGM96 
representa, com boa resolução, longos comprimentos de onda, o que basicamente significa grau e ordem 36 a 72. Daí ter-se optado por um valor intermediário $(n=m=50)$. A definição do raio em $4^{\circ}$ é uma consequiência da opção anterior. A influência dos curtos comprimentos de onda acima do grau e ordem 50 é restrita ao limite de $4^{\mathrm{o}}$;

$\mathrm{O}$ fato da modificação de L. Wong e R. Gore resultar num geóide com menor diferença quando comparado com os pontos DOP e GPS é considerado inesperado. Segundo Vanicek P. e Sjoberg L. E. (1991) e Featherstone W. E. et. al (1998) a modificação de Vanicek-Kleusberg resultaria em um menor erro de truncamento do que a modificação L. Wong e R. Gore. Já a modificação de Featherstone-Vanicek, Featherstone W. E. et al (1998) prova, através da segunda identidade de Green, que esta produziria um geóide com menor erro de truncamento do que L. Wong e R. Gore. Imagina-se que erros sistemáticos associado ao nivelamento geométrico das RNs, as quais foram usadas como referência, possa ter uma tendência a minimizar as diferenças para a modificação de L. Wong e R. Gore. Mas há incertezas que não permitem tirar conclusões definitivas. A comparação com os pontos DOP e GPS servem para indicar tendências;

Apesar do experimento FFT-WG tenha resultado numa menor diferença padrão em relação aos pontos DOP e GPS, optou-se, por razões teóricas, por um modelo geoidal calculado através da FFT com a modificação da função de Stokes proposta por Featherstone W. E. et. al. (1998). O cálculo foi feito num retângulo que envolve o Estado de São Paulo, limitado pelas latitudes de $-19^{\circ}$ a $-26^{\circ}$ SUL e pelas longitudes de $-54^{\circ}$ a $-44^{\circ}$ OESTE. A Figura 5.1 mostra o modelo geoidal SPGEOID2002 obtido para o Estado de São Paulo. Este modelo tem diferenças absolutas da ordem de 0,80 m, quando comparado com os pontos GPS e diferença relativa da ordem de $0,4 \mathrm{~cm} / \mathrm{Km}$ ou $4 \mathrm{ppm}$. As curvas de iso-valores do modelo geoidal SPGEOID2002, Figura 5.1, estão eqüidistantes de $0,20 \mathrm{~m}$;

Para aplicações de engenharia é recomendado o uso do modelo através de determinações relativas. 


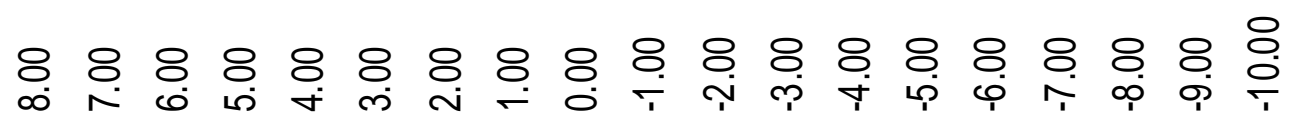

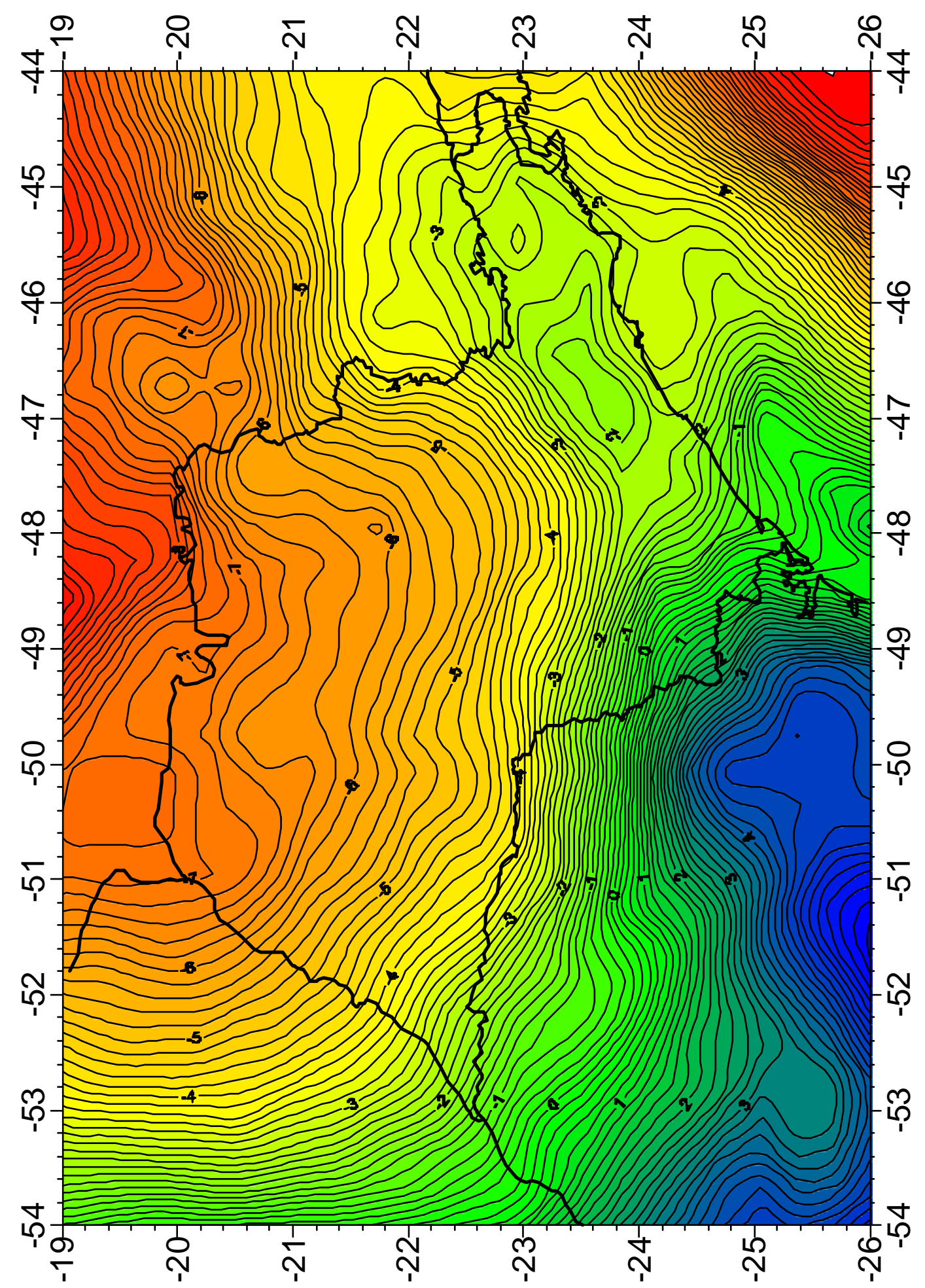

Figura 5.1 - Modelo Geoidal SPGEOID2002 para o Estado de São Paulo 


\section{REFERÊNCIAS BIBLIOGRÁFICAS}

ANDERSEN, O. B.; KNUDSEN P.; TRIMMER R. The KMS99 global marine gravity field from ERS and GEOSAT altimetry. In: ERS-Envisat Symposium. Gothenburg, Sweden. 2000.

BLITZKOW D. A combinação de diferentes tipos de dados na determinação das alturas geoidais. 1986. 91p. Tese (Doutorado) - Instituto Astronômico e Geofísico, Universidade de São Paulo. São Paulo.

BLITZKOW D. O Problema de Valor de Contorno da Geodésia - Resultados Práticos para a América do Sul. 1996. 81p. Tese (Livre Docência) - Escola Politécnica, Universidade de São Paulo. São Paulo.

FEATHERSTONE, W. E.; EVANS, J. D.; OLliVER, J. G. A Meissl-modified Vanicek and Kleusberg kernel to reduce the truncation error in gravimetric geoid computations. Journal of Geodesy, Berlin, No. 72, p. 154-160, 1998.

FORSBERG, R.; SIDERIS, M. G. Geoid computations by multi-banding spherical FFT approach. Manuscripta Geodaetica, Berlin, Vol. 18, No. 2 p. 82-90, 1993.

PAUL, MK. A Method of evaluating the truncation error coefficients for geoidal height. Bulletin Geodesique, Berlin, No. 47. p. 413-425, 1973.

RAPP, R. H; NEREM, R. S. A joint GSFC/DMA project for improving the model of the Earth's gravitational field. In: Joint Symposium of the IGC/ICG. Graz, 1994.

SIDERIS, M. G. Geoid Determination by FFT Techniques. In: International School for the Determination and Use of the Geoid. Milan. Italy, 1994.

SMITH, A.; MILBERT, D. G. (1997). Evaluation of the EGM96 Model of the Geopotential in the United States. Bulletin of International Geoid Service. Milan, Italy, No. 6, 1997. Disponível em <http://ngs.noaa.gov/PUBS_LIB/egm96.html>, Acesso em 30 jun.2000.

VANICEK, P.; SJOBER, L. E. Reformulation of Stokes's theory for higher than second-degree reference field and modification of integration kernels. Journal of Geophysical Research, New York. Vol. 96, No. B4, p. 6529-6539, 1991.

VANICEK, P.; FEATHERSTONE, W. E. Performance of three types of Stokes's kernel in the combined solution for the geoid. Journal of Geodesy, Berlin. Vol. 72, No. 12, p. 684-697, 1998.

VANICEK, P.; KLEUSBERG, A. The Canadian geoid - Stokesian approach. Manuscripta Geodaetica, Berlin. Vol 12, No. 5, p. 86-98, 1987.

WONG L.; GORE, R. Accuracy of Geoid Heights from Modified Stokes Kernels. Geophysical Journal Research. New York, Vol. 18, No.1, p. 81-91, 1969. 